\title{
SOME REMARKS ON THE RELATIVE GENUS FIELDS
}

\author{
By KoICHI TAKASE
}

\section{$\S 1$. Introduction.}

Let $k$ be a finite algebraic number field and $K$ its finite extension. We denote by $K^{*}$ the maximal abelian extension of $k$ such that the composite field $K^{*} K$ is unramified over $K$ at all the finite or infinite primes, and the field $K^{*} K$ is called the genus field of $K$ with respect of $k$. (If $K^{*}$ were defined as the maximal abelian extension of $k$ such that $K^{*} K$ was unramified over $K$ at all the finite primes, the field $K^{*} K$ was called the narrow genus field of $K$. We do not treat the narrow genus field in this paper.)

The field $K^{*}$ is explicitly determined when $k$ is the rational number field (see M. Ishida [5], [6] or M. Bhaskaran [1]). In $\S 3$ of this paper we discuss the fundamental structure of $K^{*}$ for general $k$. In $\S 4$ we treat, as an example, the case of $k=$ quadratic field of class number one in which 2 remains prime and $(K: k)=2$.

In $\S 5$ we prove the following theorem; let $k$ be a finite algebraic number field of class number one, $G$ any finite abelian group, and $m$ a positive integer such that $\operatorname{ex}(G) \mid m$ and $m \|\left. G\right|^{\infty}$. Then there exist infinitely many cyclic extensions $F$ of $k$ of degree $m$ such that

$$
C_{F} / C_{F^{\prime}}^{1-\sigma} \cong G\left(F^{*} / F\right) \cong G .
$$

This paper contains the author's master thesis at Tokyo Institute of Technology (1981, March).

\section{$\S 2$. Definitions.}

Let $k$ be a finite algebraic number field and $K$ its finite extension. We denote by $K^{*}$ the maximal abelian extension of $k$ such that $K^{*} K$ is unramified over $K$ at all the finite or infinite primes. By the class field theory, $K^{*}$ is the maximal abelian extension of $k$ in the Hilbert class field of $K$, and $K^{*} \cap K$ is the maximal abelian extension of $k$ in $K$. Throughout this paper the following notations are used;

$O_{k}:$ the integer ring of $k$

$U_{k}$ : the unit group of $k$

Received November 5, 1981 
$\phi(\mathfrak{a}):$ the Euler function of $k$

$U_{k}(\mathfrak{a})=\left\{\varepsilon \in U_{k} \mid \varepsilon \equiv 1(\bmod \mathfrak{a})\right\}$, for an integral ideal $\mathfrak{a}$ of $k$

$k_{\mathfrak{p}}$ : the completion of $k$ at a finite or infinite prime $\mathfrak{p}$ of $k$

$k_{A}^{\times}$: the idele group of $k$ into which we embed $k^{\times}$and $k_{p}^{\times}$in usual way

$k^{(1)}$ the Hilbert class field of $k$

$G(K / k)$ : the Galois group of Galois extension $K / k$

$\mathfrak{f}(K / k)$ : the conductor of abelian extension $K / k$.

\section{$\S 3$. Structure of genus field.}

Let $k$ be a finite algebraic number field, $K$ its finite extension, and fix them. For a finite prime $\mathfrak{p}$ of $k$, we put

$$
\begin{aligned}
& \mathfrak{p}=\mathfrak{P}_{1}^{e_{1}} \cdots \mathfrak{P}_{r}^{e_{r}}\left(\mathfrak{P}_{1}, \cdots, \mathfrak{P}_{r}: \text { distinct primes of } K, e_{\jmath}>0\right) \\
& e_{K}(\mathfrak{p})=\text { g.c.d. }\left\{e_{1}, \cdots, e_{r}\right\}, g_{k}(\mathfrak{p})=\phi(\mathfrak{p}) /\left(U_{k}: U_{k}(\mathfrak{p})\right), \\
& d_{K}(\mathfrak{p})=\text { g.c.d. }\left\{e_{K}(\mathfrak{p}), g_{k}(\mathfrak{p})\right\} .
\end{aligned}
$$

Let $S(\mathfrak{p})$ be the ray class field modulo $\mathfrak{p}$ of $k$. Then $S(\mathfrak{p}) / k^{(1)}$ is a cyclic extension of degree $g_{k}(\mathfrak{p})$, and we put

$k(\mathfrak{p})$ : unique intermediate field of $S(\mathfrak{p}) / k^{(1)}$ such that $\left(k(\mathfrak{p}): k^{(1)}\right)=d_{K}(\mathfrak{p})$.

Then we have

LEMma 1. $k(\mathfrak{p}) \subset K^{*}$ for any finite prime $\mathfrak{p}$ of $k$.

Proof. This lemma is proved in [4]. Another proof using Abhyanker's lemma is given in [3].

We define two subfield $K_{1}^{*}$ and $K_{2}^{*}$ of $K^{*}$ by

$$
K_{1}^{*}=\prod_{\mathfrak{p}} k(\mathfrak{p}): \text { composite field, } K_{2}^{*}=\bigcap_{\mathfrak{p}} T(\mathfrak{p}) \text {, }
$$

where $\mathfrak{p}$ runs over all finite primes of $k$ such that $e_{K}(\mathfrak{p}) \mid g_{k}(\mathfrak{p})$, and $T(\mathfrak{p})$ is the inertia field of $\mathfrak{p}$ in $K^{*} / k$. Notice that, for distinct finite primes $\mathfrak{p}_{1}, \cdots, \mathfrak{p}_{r}$ of $k$, the fields $k\left(\mathfrak{p}_{1}\right), \cdots, k\left(\mathfrak{p}_{r}\right)$ are linearly disjoint over $k^{(1)}$. Then we have

THEOREM 2.

$$
K_{1}^{*} \cap K_{2}^{*}=k^{(1)}, \quad K^{*}=K_{1}^{*} K_{2}^{*} .
$$

Proof. Because the primes of $k$ which are ramified in $K_{1}^{*}$ are unramified in $K_{2}^{*}$, the field $K_{1}^{*} \cap K_{2}^{*}$ is an unramified abelian extension of $k$. Hence we have $K_{1}^{*} \cap K_{2}^{*}=k^{(1)}$, since $K_{1}^{*} \cap K_{2}^{*}$ contains $k^{(1)}$.

Because $K^{*} K / K$ is unramified, we have $e_{K^{*}}(\mathfrak{p}) \mid e_{K}(\mathfrak{p})$ for any finite prime $\mathfrak{p}$ of $k$. Then we have the following inequalities from which the equality 
$K^{*}=K_{1}^{*} K_{2}^{*}$ follows;

$$
\begin{aligned}
\left(K_{1}^{*}: k^{(1)}\right)=\left(K_{1}^{*} K_{2}^{*}: K_{2}^{*}\right) \leqq\left(K^{*}: K_{2}^{*}\right) & \leqq \prod_{p}\left(K^{*}: T(\mathfrak{p})\right) \\
& \leqq \prod_{\mathfrak{p}}\left(k(\mathfrak{p}): k^{(1)}\right)=\left(K_{1}^{*}: k^{(1)}\right)
\end{aligned}
$$

On the conductor of abelian extension $K^{*} / k$, we have the following theorem:

THEOREM 3. Suppose that $K$ is a normal extension of $k$. Then $\mathfrak{f}\left(K^{*} / k\right)$ $=\mathrm{f}\left(K^{*} \cap K / k\right)$. (Notıce that the field $K^{*} \cap K$ is the maximal abelian extension of $k$ in $K$.)

Proof. Put $U=\prod_{\mathfrak{B}} U_{\mathfrak{B}}$ the unit idele group of $K$, where $\mathfrak{P}$ runs over all finite or infinite primes of $K$ and $U_{\mathfrak{B}}$ is the unit group of $K_{\mathfrak{P}}$. Then, by the class field theory, we have

$K^{*}=$ the class field of $k$ corresponding to $k^{\times} N_{K / k} U$,

$K^{*} \cap K=$ the class field of $k$ corresponding to $k^{\times} N_{K / k} K_{A}^{\times}$.

Since $K$ is normal over $k$, we have

$$
N_{K / k} U=\prod_{\mathfrak{p}} N_{\mathfrak{P} / \mathfrak{p}} U_{\mathfrak{B}}, \quad N_{K / k} K_{A}^{\times}=k_{A}^{\times} \cap \prod_{\mathfrak{p}} N_{\mathfrak{B} / \mathfrak{p}} K_{\mathfrak{B}}^{\times}
$$

where $\mathfrak{p}$ runs over all the finite or infinite primes of $k, \mathfrak{P}$ is any one of the primes of $K$ lying over $\mathfrak{p}$, and $N_{\mathfrak{P} / \mathfrak{p}}$ is the norm from $K_{\mathfrak{P}}$ to $k_{\mathfrak{p}}$. Because the inverse image of $U_{\mathfrak{p}}$ by $N_{\mathfrak{B} / \mathfrak{p}}$ is contained in $U_{\mathfrak{B}}$, we have $\mathfrak{f}\left(K^{*} / k\right)=\mathfrak{f}\left(K^{*} \cap K / k\right)$.

COROLlary 4. Suppose that $K$ is a normal extension of $k$. Then $K_{*}=K_{1}^{*}$ if and only if $K^{*} \cap K / k$ is unramified at the infinite primes and $e_{K}(\mathfrak{p}) \mid g_{k}(\mathfrak{p})$ for any finte prime $\mathfrak{p}$ of $k$ ramified in $K^{*} \cap K$.

Proof. Let $\mathfrak{p}_{1}, \cdots, \mathfrak{p}_{r}$ be the finite primes of $k$ such that $e_{K}\left(\mathfrak{p}_{j}\right) \mid g_{k}\left(\mathfrak{p}_{j}\right)$ and $e_{K}\left(\mathfrak{p}_{j}\right)>1$. Then we have $\mathfrak{f}\left(K_{1}^{*} / k\right)=\mathfrak{p}_{1} \cdots \mathfrak{p}_{r}$. Because $\mathfrak{f}\left(K_{1}^{*} / k\right)$ and $\mathfrak{f}\left(K_{2}^{*} / k\right)$ are relatively prime and $K^{*}$ is the composite field of $K_{1}^{*}$ and $K_{2}^{*}$, we have $\mathfrak{f}\left(K^{*} k\right)$ $=\mathfrak{f}\left(K_{1}^{*} / k\right) \mathfrak{f}\left(K_{2}^{*} / k\right)$. Because $K_{1}^{*} \cap K_{2}^{*}$ is equal to $k^{(1)}$ and $K_{2}^{*}$ contains $k^{(1)}, K^{*}=K_{1}^{*}$ if and only if $\mathfrak{f}\left(K_{2}^{*} / k\right)=1$, that is, if and onlf if $\mathfrak{f}\left(K^{*} / k\right) \mid \mathfrak{f}\left(K_{1}^{*} / k\right)$. Hence, because of Theorem $3, K^{*}=K_{1}^{*}$ if and only if $\mathfrak{f}\left(K^{*} \cap K / k\right) \mid \mathfrak{f}\left(K_{1}^{*} / k\right)$, and only-ifpart of the assertion is proved.

If $K^{*} \cap K / k$ is unramified at the infinite primes and $e_{K}(\mathfrak{p}) \mid g_{k}(\mathfrak{p})$ for any finite prime $\mathfrak{p}$ of $k$ which is ramified in $K^{*} \cap K / k, K^{*} \cap K$ is tamely ramified over $k$ at the finite primes and hence $\mathfrak{f}\left(K^{*} \cap K / k\right)$ is square-free. Because the set $\left\{\mathfrak{p}_{1}, \cdots, \mathfrak{p}_{r}\right\}$ includes the prime factors of $\mathfrak{f}\left(K^{*} \cap K / k\right)$ by hypothesis, we have $\mathfrak{f}\left(K^{*} \cap K / k\right) \mid \mathfrak{f}\left(K_{1}^{*} / k\right)$.

Proposition 5. Suppose that $K$ is an abelian extension of $k$ which is unramified over $k$ at the infinite primes and that there exists ouly one finite prime 
$\mathfrak{p}$ of $k$ such that $e_{K}(\mathfrak{p}) \chi g_{k}(\mathfrak{p})$. Then we have

$K^{*}=K_{1}^{*} K, K_{1}^{*} \cap K=$ the inertıa field of $\mathfrak{p}$ in $K / k$.

Proof. Since $\mathfrak{p}$ is unique prime of $k$ which may be ramified in $K_{2}^{*}, \mathfrak{p}$ is totally ramified in $K_{2}^{*} / k^{(1)}$. Because $K^{*} K$ is unramified over $K$, we have $\left(K_{2}^{*}: k^{(1)}\right) \mid e_{K}(\mathfrak{p})$. As $\mathfrak{p}$ is unramified in $K_{1}^{*} \cap K$, we have

$K_{1}^{*} \cap K \subset T=$ the inertia field of $\mathfrak{p}$ in $K / k$.

Therefore we have the following inequalities from which our assertion follows;

$$
\begin{aligned}
\left(K_{1}^{*}: k\right) & =\left(K_{1}^{*} K: K\right)\left(K_{1}^{*} \cap K: k\right) \\
& \leqq\left(K^{*}: K\right)(T: k) \\
& =\left(K_{1}^{*}: k^{(1)}\right)\left(K_{2}^{*}: k^{(1)}\right)\left(k^{(1)}: k\right) /(K . T) \leqq\left(K_{1}^{*}: k\right) .
\end{aligned}
$$

In Proposition 5 the uniqueness of prime $\mathfrak{p}$ of $k$ such that $e_{K}(\mathfrak{p}) \chi g_{k}(\mathfrak{p})$ is indispensable as the following example shows:

ExAmple. Put $k=Q(\sqrt{-11})$. The polynomial $f(X)=X^{3}-3 X-1$ is irreducible over $k$. Let $\alpha$ be a root of $f(X)=0$ and put $K=k(\alpha)$. Then $K$ is a cyclic extension of $k$ of degree 3 and the relative discriminant of $K$ over $k$ is $D(K / k)=3^{4}$. The prime factors $D(K / k)$ in $k$ are $1+\omega$ and $\omega$ where $\omega=(1+\sqrt{-11}) / 2$. Since $g_{k}(1+\omega)=g_{k}(\omega)=1, e_{K}(1+\omega)=e_{K}(\omega)=3$, we have

$$
K_{1}^{*}=k \text {. }
$$

On the other hand, by the genus number formula proved in [2], we have

$$
\left(K^{*}: k\right)=9 \text {. }
$$

For the latter use, we prove the following lemma:

Lemma 6. Let $L$ and $M$ be finite extension of $k$ such that $\left(L_{1}: k\right)$ and $(M: k)$ are relatively prime. Then we have $(L M)^{*}=L^{*} M^{*}$.

Proof. Put $K=L M$. The inclusion $L^{*} M^{*} \subset K^{*}$ is obvious. We have to prove that any finite abelian extension $F$ of $k$ such that $F K$ is unramified over $K$ is contained in $L^{*} M^{*}$. We can suppose that $(F: k)$ is a power of a rational prime $l$ and the $F$ is ramified over $k$. Then, as $F K$ is unramified over $K$, we have $l \mid(K: k)$ and hence $l \mid(L: k)$ or $l \mid(M: k)$. Suppose $l \mid(L: k)$. Since $(F L: L)=(F: F \cap L)$ is a power of $l$, the ramification index in $F L / L$ of the finite primes of $L$ are power of $l$. Because $F K$ is unramified over $K$ and $l \chi(M: k)=(K: L), F L$ is unramified over $L$ and so $F \subset L^{*} \subset L^{*} M^{*}$. 


\section{§4. Examples.}

Let $k$ be a finite algebraic number field of class number one in which 2 remains prime. Let $(k: Q)=n$ and $\left\{\omega_{j}^{2}\left(\jmath=1,2, \cdots, 2^{n}-1\right)\right\}$ be a system of complete representatives of the squares of the multiplicative group of $O_{k} /(4)$ (its order is easily shown to be $2^{n}-1$ ). Let $m$ be a square-free integer of $k$ and put $K=k(\sqrt{m})$. We define an integer $\theta$ of $K$ by

$$
\theta=\left\{\begin{array}{cl}
\left(\omega_{j}+\sqrt{m}\right) / 2: & m \equiv \omega_{j}^{2}(\bmod 4) \text { for some } j \\
\sqrt{m}: & \text { otherwise. }
\end{array}\right.
$$

Then we have

LEMma 7. $O_{K}$ is a free $O_{k}$-module with base $\{1, \theta\}$, and the relative discriminant of $K$ over $k$ is given by

$$
D(K / k)=\left\{\begin{aligned}
m: m \equiv \omega_{j}^{2}(\bmod 4) \text { for some } j \\
4 m: \text { otherwise. }
\end{aligned}\right.
$$

Proof. We use the following fact; for integers $a, b$ and $c$ of $k$, the equation

$$
a^{2}-b^{2} c \equiv 0(\bmod 4)
$$

is equivalent to $a \equiv b \omega_{j}(\bmod 2)$ if $c \equiv \omega_{j}^{2}(\bmod 4)$ for some $j$, and to $a \equiv b \equiv 0$ $(\bmod 2)$ if $c \not \equiv \omega_{j}^{2}(\bmod 4)$ for any $j$. Because $m$ is a square-free integer of $k$, we have

$$
\begin{aligned}
O_{K} & =\left\{a+b \sqrt{m} \mid a, b \in k \text { such that } 2 a \in O_{k}, a^{2}-b^{2} m \in O_{k}\right\} \\
& =\left\{(a+b \sqrt{m}) / 2 \mid a, b \in O_{k} \text { such that } a^{2}-b^{2} m \equiv 0(\bmod 4)\right\} .
\end{aligned}
$$

If $m \equiv \omega_{J}^{2}(\bmod 4)$ for some $\jmath$, we have by above remark

$$
\begin{aligned}
O_{K} & =\left\{\left(a-b \omega_{\jmath}\right) / 2+b\left(\omega_{j}+\sqrt{m}\right) / 2 \mid a, b \in O_{k} \text { such that } a \equiv b \omega_{\jmath}(\bmod 2)\right\} \\
& =\left\{a+b \theta \mid a, b \in O_{k}\right\} .
\end{aligned}
$$

If $m \neq \omega_{j}^{2}(\bmod 4)$ for any $j$, we have

$$
O_{K}=\left\{a+b \sqrt{m} \mid a, b \in O_{k}\right\} .
$$

We have

$$
K_{1}^{*}=\prod_{\mathfrak{p}} k(\mathfrak{p}), \quad K_{2}^{*}=\bigcap_{\mathfrak{p}} T(\mathfrak{p})
$$

where $\mathfrak{p}$ runs over the prime factors of $D(K / k)$ in $k$ such that $2 \mid g_{k}(\mathfrak{p})$, and $T(\mathfrak{p})$ is the inertia field of $\mathfrak{p}$ in $K^{*} / k$. For a prime factor $\mathfrak{p}$ of $D(K / k)$ in $k$ such that $2 \mid g_{k}(\mathfrak{p}), k(\mathfrak{p})$ is a quadratic extension of $k$, and by Lemma $7, k(\mathfrak{p})=k(\sqrt{\pi})$ where $\pi$ is a generator of $\mathfrak{p}$ such that $\pi \equiv \omega_{j}^{2}(\bmod 4)$ for some $\jmath$ and satisfies conditions on its signature (if necessary). 
We treat more explicitly the case of $k=$ quadratic field below.

1) Let $k$ be a imaginary quadratic field of class number one in which 2 remains prime, that is, $k=Q(\sqrt{D})$ where $D=-3,-11,-19,-43,-67,-163$, and put $\omega=(-1+\sqrt{D}) / 2$. Then $\{a+b \omega \mid a, b=0,1,2,3\}$ is a system of complete representatives of $O_{k}$ modulo 4 . There are only three representatives which are prime to 2 and are congruent modulo 4 to squares, and they are named as in the following table:

\begin{tabular}{c|c|c|c}
\hline$D$ & $\omega_{1}$ & $\omega_{2}$ & $\omega_{3}$ \\
\hline$-3,-19$ & 1 & $\omega \equiv(1+\omega)^{2}$ & $3+3 \omega \equiv \omega^{2}$ \\
$-67,-163$ & 1 & $2+\omega \equiv(1+\omega)^{2}$ & $1+3 \omega \equiv \omega^{2}$
\end{tabular}

Let $m$ be a square-free integer of $k$ and put $K=k(\sqrt{m})$. Let $\theta$ be an integer of $K$ defined by

$$
\theta=\left\{\begin{array}{cll}
(1+\sqrt{m}) / 2 & : & m \equiv \omega_{1}(\bmod 4) \\
(1+\omega+\sqrt{m}) / 2: & m \equiv \omega_{2}(\bmod 4) \\
(\omega+\sqrt{m}) / 2 & : & m \equiv \omega_{3}(\bmod 4) \\
\sqrt{m} & : & \text { otherwise }
\end{array}\right.
$$

Then, by Lemma $7, O_{k}$ is a free $O_{k}$-module with base $\{1, \theta\}$ and the relative discriminant of $K$ over $k$ is given by

$$
D(K / k)=\left\{\begin{aligned}
m: & m \equiv \omega_{1}, \omega_{2}, \omega_{3}(\bmod 4) \\
4 m: & \text { otherwise }
\end{aligned}\right.
$$

For a finite prime $\mathfrak{p}$ of $k$, we have

$$
k(\mathfrak{p})=\left\{\begin{aligned}
k(\sqrt{\pi}): & \text { if } \mathfrak{p}=(\pi) \text { where } \pi \equiv \omega_{1}, \omega_{2}, \omega_{3}(\bmod 4) \\
k: & \text { otherwise. }
\end{aligned}\right.
$$

ExAmple 1. Put $k=Q(\sqrt{ }-11), K=k(\sqrt{ } 5)$. Because 5 is a square-free integer of $k$ and $5 \equiv \omega_{1}(\bmod 4)$, we have $D(K / k)=5$. The prime factors of $D(K / k)$ in $k$ are $1-\omega$ and $2+\omega$. Since $g_{k}(1-\omega)=g_{k}(2+\omega)=2$ and $1-\omega \equiv \omega_{3}$ $(\bmod 4), 2+\omega \equiv \omega_{2}(\bmod 4)$, we have by Corollary 4

$$
K^{*}=K_{1}^{*}=k(\sqrt{1-\omega}, \sqrt{2+\omega}) .
$$

ExAmple 2. Put $k=Q(\sqrt{-3}), K=k(\sqrt{ } 26)$. Because 26 is a square-free integer of $k$ and $26 \neq \omega_{1}, \omega_{2}, \omega_{3}(\bmod 4)$, we have $D(K / k)=2^{3} 13$. The prime 
factors of $D(K / k)$ in $k$ are $2,3-\omega$ and $4+\omega$. Since $g_{k}(2)=1, g_{k}(3-\omega)=g_{k}(4+\omega)$ $=2$ and $3-\omega \equiv \omega_{3}(\bmod 4), 4+\omega \equiv \omega_{2}(\bmod 4)$, we have by Proposition 5

$$
K^{*}=K_{1}^{*} K=k(\sqrt{26}, \sqrt{3-\omega}, \sqrt{4+\omega}) .
$$

2) There are ten real quadratic field of discriminant less than 100 of class number one in which 2 remains prime, that is, $Q(\sqrt{D})$ where $D=5,13,21,29$, $37,53,61,69,77,93$. Let $k$ be one of the ten real quadratic fields and put $\omega=(-1+\sqrt{ } D) / 2$. Then $\{a+b \omega \mid a, b=0,1,2,3\}$ is a system of complete representatives of $O_{k}$ modulo 4 . There are only three representatives of $O_{k}$ modulo 4 which are prime to 2 and are congruent modulo 4 to squares, and they are named as in the following table

\begin{tabular}{c|c|c|c}
\hline & & $\omega_{1}$ & $\omega_{3}$ \\
\hline $\begin{array}{l}5,21,37 \\
53,69\end{array}$ & 1 & $2+\omega \equiv(1+\omega)^{2}$ & $1+3 \omega \equiv \omega^{2}$ \\
\hline $\begin{array}{l}13,29,61 \\
77,93\end{array}$ & 1 & $\omega \equiv(1+\omega)^{2}$ & $3+3 \omega \equiv \omega^{2}$
\end{tabular}

Let $m$ be a square-free integer of $k$ and put $K=k(\sqrt{ } m)$. Let $\theta$ be an integer of $K$ defined by

$$
\theta=\left\{\begin{array}{cll}
(1+\sqrt{m}) / 2: & m \equiv \omega_{1}(\bmod 4) \\
(1+\omega+\sqrt{m}) / 2: & m \equiv \omega_{2}(\bmod 4) \\
(\omega+\sqrt{m}) / 2: & m \equiv \omega_{3}(\bmod 4) \\
\sqrt{m} & : & \text { otherwise. }
\end{array}\right.
$$

Then, by Lemma $7, O_{K}$ is a free $O_{k}$-module with base $\{1, \theta\}$ and the relative discriminant of $K$ over $k$ is given by

$$
D(K / k)=\left\{\begin{aligned}
m: & m \equiv \omega_{1}, \omega_{2}, \omega_{3}(\bmod 4) \\
4 m: & \text { otherwise }
\end{aligned}\right.
$$

For a finite prime $\mathfrak{p}$ of $k$, we have

$$
k(\mathfrak{p})=\left\{\begin{aligned}
k(\sqrt{\pi}): & \text { if } \mathfrak{p}=(\pi) \text { where } \pi \equiv \omega_{1}, \omega_{2}, \omega_{3}(\bmod 4) \text { and } \pi \geqq 0 \\
k: & \text { otherwise }
\end{aligned}\right.
$$

where $\pi \geqq 0$ means that $\pi$ is totally positive.

ExAmple 3. Put $k=Q(\sqrt{ } 13), K=k(\sqrt{ } 53)$. Because 53 is a square-free integer of $k$ and $53 \equiv \omega_{1}(\bmod 4)$, we have $D(K / k)=53$. The prime factors of $D(K / k)$ in $k$ are $7-\omega$ and $8+\omega$. Since $g_{k}(7-\omega)=g_{k}(8+\omega)=2$ (see the tables at the end of this $\S)$, and $7-\omega \equiv \omega_{3}(\bmod 4), 8+\omega \equiv \omega_{2}(\bmod 4), 7-\omega \geqq 0,8+\omega \geqq 0$, 
we have by Corollary 4

$$
K^{*}=K_{1}^{*}=k(\sqrt{7-\omega}, \sqrt{8+\omega}) .
$$

EXAMPLE 4. Put $k=Q(\sqrt{29}), K=k(\sqrt{ } \overline{10})$. Because 10 is a sequare-free integer of $k$ and $10 \neq \omega_{1}, \omega_{2}, \omega_{3}(\bmod 4)$, we have $D(K / k)=2^{3} 5$. The prime factors of $D(K / k)$ in $k$ are $2,4+\omega$, and $3-\omega$. Since $g_{k}(2)=1, g_{k}(4+\omega)$ $=g_{k}(3-\omega)=2$, and $4+\omega \equiv \omega_{2}(\bmod 4), 3-\omega \equiv \omega_{3}(\bmod 4), 4+\omega \geqq 0,3-\omega \geqq 0$, we have by Proposition 5

$$
K^{*}=K_{1}^{*} K=k(\sqrt{10}, \sqrt{4+\omega}, \sqrt{3-\omega}) .
$$

ExAmple 5. Put $k=Q(\sqrt{ } 53), K=k(\sqrt{221})$. Because 221 is a square-free integer of $k$ and $221 \equiv \omega_{1}(\bmod 4)$, we have $D(K / k)=13 \cdot 17$. The prime factors of $D(K / k)$ in $k$ are $13+3 \omega, 17+4 \omega, 5-\omega$, and $6+\omega$. Since $g_{k}(13+3 \omega)=g_{k}(17+4 \omega)$ $=g_{k}(5-\omega)=g_{k}(6+\omega)=2$, and $13+3 \omega \equiv \omega_{3}(\bmod 4), 17+4 \omega \equiv \omega_{1}(\bmod 4), 5-\omega \equiv \omega_{3}$ $(\bmod 4), 6+\omega \equiv \omega_{2}(\bmod 4), 13+3 \omega \geqq 0,17+4 \omega \geqq 0,5-\omega \geqq 0,6+\omega \geqq 0$, we have

$$
K^{*}=K_{1}^{*}=k(\sqrt{13+3 \omega}, \sqrt{17+4 \omega}, \sqrt{5-\omega}, \sqrt{6+\omega}) .
$$

Let $L$ be the genus field of $K$ with respect to the rational number field, that is, the maximal abelian extension of $Q$ such that $K L / K$ is unramified. Then we have by the genus number formula

$$
(L: Q) \leqq 2^{3} \quad \text { i. e. } \quad(L: k) \leqq 2^{2}
$$

On the other hand, we have $\left(K^{*}: k\right)=2^{4}$ and hence $L \subsetneq K^{*}$.

Tables.

Table of $g_{k}(p)$ and prime elements of $k$ above each rational primes. (Blanks mean that the rational prime remains prime in $k$.)

a) $k=Q(\sqrt{5}), \omega=(-1+\sqrt{5}) / 2$, fundamental unit $=(1+\sqrt{5}) / 2=1+\omega$

\begin{tabular}{|c|c|c|c|c|c|c|c|c|c|c|c|c|}
\hline & 2 & \multicolumn{2}{|c|}{3} & 5 & 7 & 11 & 13 & \multicolumn{2}{|c|}{17} & 19 & \multicolumn{2}{|c|}{23} \\
\hline & & $\omega$ & $1+\omega$ & & & & $1+2 \omega$ & $4-\omega$ & $5+\omega$ & & $1-3 \omega$ & $4+3 \omega$ \\
\hline$g_{k}(\mathfrak{p})$ & 1 & 1 & 1 & 2 & 3 & $3 \cdot 5$ & 3 & 1 & 1 & $3^{2}$ & 1 & 1 \\
\hline
\end{tabular}

\begin{tabular}{|c|c|c|c|c|c|c|c|c|c|c|c|c|c|}
\hline & 2 & 3 & 5 & 7 & \multicolumn{2}{|c|}{11} & 13 & 17 & \multicolumn{2}{|c|}{19} & 23 & \multicolumn{2}{|c|}{29} \\
\hline & & & $2-\omega$ & & $3-\omega$ & $4+\omega$ & & & $4-\omega$ & $5+\omega$ & & $5-\omega$ & $6+\omega$ \\
\hline$g_{k}(\mathfrak{p})$ & 1 & 1 & 1 & 3 & 1 & 1 & $2 \cdot 3$ & $2^{3}$ & 1 & 1 & 11 & 2 & 2 \\
\hline
\end{tabular}

\begin{tabular}{|c|c|c|c|c|c|c|c|c|c|}
\hline \multicolumn{2}{|c|}{31} & 37 & \multicolumn{2}{|c|}{41} & 43 & 47 & 53 & \multicolumn{2}{|c|}{59} \\
\hline $7+2 \omega$ & $5-2 \omega$ & & $6-\omega$ & $7+\omega$ & & & & $9+2 \omega$ & $7-2 \omega$ \\
\hline 1 & 1 & $2 \cdot 3^{2}$ & 1 & 1 & $3 \cdot 7$ & $3 \cdot 23$ & $2 \cdot 13$ & 1 & 1 \\
\hline
\end{tabular}

b) $k=Q(\sqrt{13}), \omega=(-1+\sqrt{13}) / 2$, fundamental unit $=(3+\sqrt{13}) / 2=2+\omega$ 


\begin{tabular}{|c|c|c|c|c|c|c|c|c|c|c|c|c|}
\hline \multicolumn{2}{|c|}{29} & \multirow[t]{2}{*}{31} & \multirow[t]{2}{*}{37} & \multirow[t]{2}{*}{41} & \multicolumn{2}{|c|}{43} & \multirow[t]{2}{*}{47} & \multicolumn{2}{|c|}{53} & \multirow[t]{2}{*}{59} & \multicolumn{2}{|c|}{61} \\
\hline $2+3 \omega$ & $1+3 \omega$ & & & & $1-4 \omega$ & $5+4 \omega$ & & $7-\omega$ & $8+\omega$ & & $8-3 \omega$ & $11+3 \omega$ \\
\hline 1 & 1 & $3 \cdot 5$ & $2 \cdot 3^{2}$ & $2^{2} \cdot 3 \cdot 5 \mid$ & 1 & 1 & 23 & 2 & 2 & $5 \cdot 29$ & 2 & 2 \\
\hline
\end{tabular}

c) $k=Q(\sqrt{29}), \omega=(-1+\sqrt{29}) / 2$, fundamental unit $=(5+\sqrt{29}) / 2=3+\omega$

\begin{tabular}{|c|c|c|c|c|c|c|c|c|c|c|c|c|c|}
\hline & 2 & 3 & \multicolumn{2}{|c|}{5} & \multicolumn{2}{|c|}{7} & 11 & \multicolumn{2}{|c|}{13} & 17 & 19 & \multicolumn{2}{|c|}{23} \\
\hline & & & $1-\omega$ & $2+\omega$ & $\omega$ & $1+\omega$ & & $4-\omega$ & $5+\omega$ & & & $5-\omega$ & $6+\omega$ \\
\hline$g_{k}(\mathfrak{p})$ & 1 & 1 & 2 & 2 & 1 & 1 & 5 & 1 & 1 & $2^{3}$ & $3^{2}$ & 1 & 1 \\
\hline
\end{tabular}

\begin{tabular}{|c|c|c|c|c|c|c|c|c|c|c|}
\hline 29 & 31 & 37 & 41 & 43 & 47 & \multicolumn{2}{|c|}{53} & \multicolumn{2}{|c|}{59} & 61 \\
\hline $1+2 \omega$ & & & & & & $5+3 \omega$ & $2-\omega$ & $1-3 \omega$ & $4+3 \omega$ & \\
\hline 7 & $3 \cdot 5$ & $2 \cdot 3^{2}$ & $2^{2} \cdot 3 \cdot 5$ & $3 \cdot 7$ & 23 & 1 & 1 & 1 & 1 & $2 \cdot 3$ \\
\hline
\end{tabular}

d) $k=Q(\sqrt{ } 37), \omega=(-1+\sqrt{ } 37) / 2$, fundamental unit $=6+\sqrt{37}=7+2 \omega$

\begin{tabular}{|c|c|c|c|c|c|c|c|c|c|c|c|c|c|}
\hline & 2 & & 3 & 5 & & & & 11 & 13 & 17 & 19 & 23 & 29 \\
\hline & & $2-\omega$ & $3+\omega$ & & $1-\omega$ & $2+\omega$ & $4-\omega$ & $5+\omega$ & & & & & \\
\hline$g_{k}(p)$ & 3 & 1 & 1 & 2 & 1 & 1 & 1 & 1 & $2 \cdot 3$ & $2^{3}$ & $3^{2}$ & 11 & $2 \cdot 5 \cdot 7$ \\
\hline 31 & 37 & 4 & 1 & 43 & 4 & & & 3 & 59 & 61 & & & \\
\hline & $1+2 \omega$ & $8+3 \omega$ & $5-3 \omega$ & & $7-\omega$ & $8+\omega$ & $4-3 \omega$ & $7+3 \omega$ & & & & & \\
\hline $3 \cdot 5$ & $3^{2}$ & 1 & 1 & $3 \cdot 7$ & 1 & 1 & 1 & 1 & 29 & $2 \cdot 3 \cdot 5$ & & & \\
\hline
\end{tabular}

e) $k=Q(\sqrt{ } 5 \overline{3}), \omega=(-1+\sqrt{ } 53) / 2$, fundamental unit $=(7+\sqrt{ } 5 \overline{3}) / 2=4+\omega$

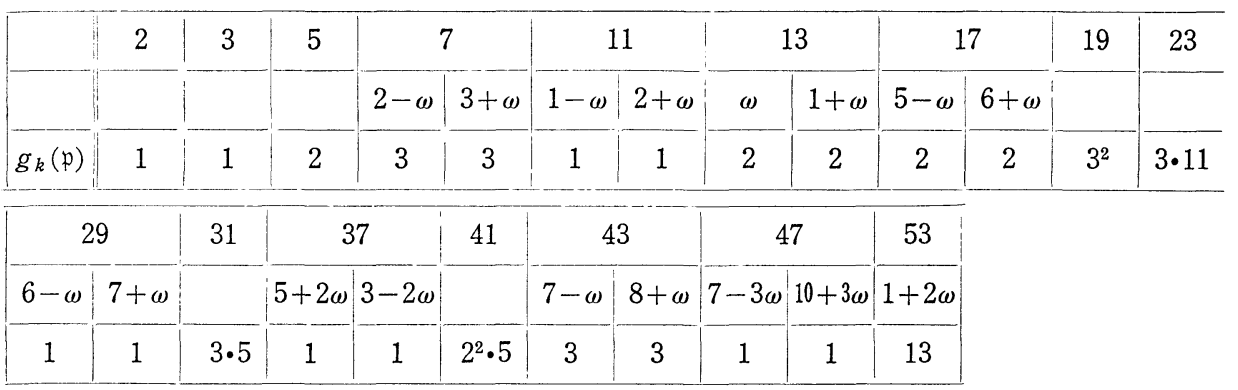

f) $k=Q(\sqrt{6} \overline{1}), \omega=(-1+\sqrt{ } \overline{1} \overline{1}) / 2$, fundamental unit $=(39+5 \sqrt{6} \overline{1}) / 2=22+5 \omega$

\begin{tabular}{|c|c|c|c|c|c|c|c|c|c|c|c|c|c|}
\hline & 2 & 3 & \multicolumn{2}{|c|}{5} & 7 & 11 & 13 & 17 & 19 & 23 \\
\hline & & $4+\omega$ & $3-\omega$ & $4-\omega$ & $5+\omega$ & & & $1-\omega$ & $2+\omega$ & & $11-3 \omega$ & $14+3 \omega$ & \\
\hline$g_{k}(\mathfrak{p})$ & 1 & 1 & 1 & 1 & 1 & 3 & 5 & $2 \cdot 3$ & $2 \cdot 3$ & $2^{3}$ & 1 & 1 & $3 \cdot 11$ \\
\hline
\end{tabular}




\begin{tabular}{|c|c|c|c|c|c|c|c|c|c|c|c|}
\hline 29 & 31 & 37 & \multicolumn{2}{|c|}{41} & 43 & 47 & 53 & 59 & 61 & 67 \\
\hline & & & $7-\omega$ & $8+\omega$ & & $11+3 \omega$ & $8-3 \omega$ & & & $1+2 \omega$ & \\
\hline $2^{2} \cdot 7$ & $3 \cdot 15$ & $2 \cdot 3^{2}$ & $2^{2}$ & $2^{2}$ & $3 \cdot 7$ & 1 & 1 & $2 \cdot 3 \cdot 13$ & $5 \cdot 29$ & $3 \cdot 5$ & $?$ \\
\hline
\end{tabular}

g) $k=Q(\sqrt{2} \overline{1}), \omega=(-1+\sqrt{ } 21) / 2$, fundamental unit $=(5+\sqrt{ } 2 \overline{1}) / 2=3+\omega$

\begin{tabular}{|c|c|c|c|c|c|c|c|c|c|c|c|c|c|}
\hline & 2 & 3 & \multicolumn{2}{|c|}{5} & 7 & 11 & 13 & 17 & 19 & 23 & 29 & 31 \\
\hline & & $1-\omega$ & $1+\omega$ & $\omega$ & $3-\omega$ & & & $3+2 \omega$ & $1-2 \omega$ & & & & \\
\hline$g_{k}(p)$ & 1 & 1 & 1 & 1 & 3 & $2 \cdot 5$ & $2 \cdot 3$ & 1 & 1 & $2^{2} \cdot 3^{2}$ & $2 \cdot 3 \cdot 112^{2} \cdot 3 \cdot 7$ & $2^{2} \cdot 3 \cdot 5$ \\
\hline
\end{tabular}

\begin{tabular}{|c|c|c|c|c|c|c|c|c|c|c|c|}
\hline \multicolumn{2}{|c|}{37} & \multicolumn{2}{|c|}{41} & \multicolumn{2}{|c|}{43} & \multicolumn{2}{|c|}{47} & 53 & \multicolumn{2}{|r|}{59} & 61 \\
\hline $6-\omega$ & $7+\omega$ & $1-3 \omega$ & $4+3 \omega$ & $9+2 \omega$ & $7-2 \omega$ & $2+3 \omega$ & $1+3 \omega$ & & $7+4 \omega$ & $|3-4 \omega|$ & \\
\hline 2 & 2 & 1 & 1 & 1 & 1 & 1 & 1 & $2^{2} \cdot 13$ & 1 & 1 & $2^{2} \cdot 3 \cdot 5$ \\
\hline
\end{tabular}

h) $k=Q(\sqrt{69}), \omega=(-1+\sqrt{ } 6 \overline{9}) / 2$, fundamental unit $=(25+3 \sqrt{ } 6 \overline{9}) / 2=4+3 \omega$

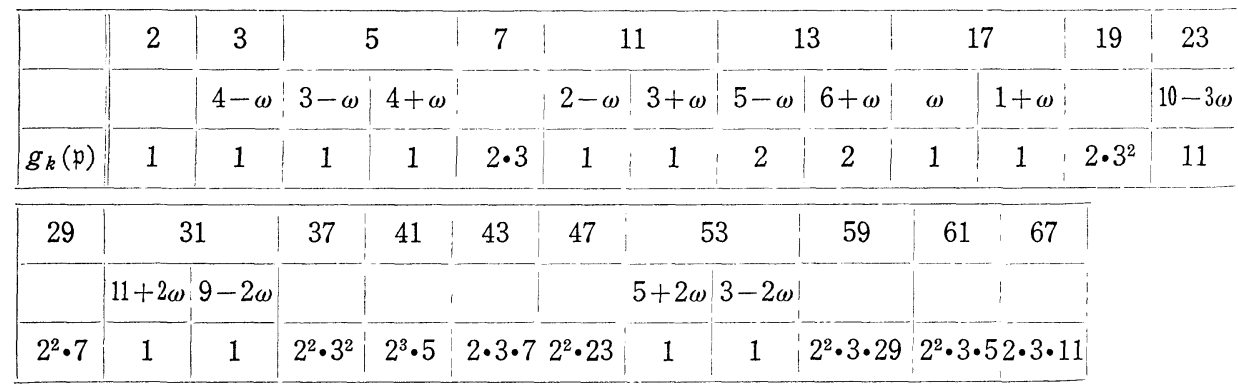

i) $k=Q(\sqrt{77}), \omega=(-1+\sqrt{77}) / 2$, fundamental unit $=(9+\sqrt{ } 77) / 2=5+\omega$

\begin{tabular}{|c|c|c|c|c|c|c|c|c|c|c|c|c|c|}
\hline & 2 & 3 & 5 & 7 & 11 & | & 3 & \multicolumn{2}{|c|}{17} & \multicolumn{2}{|c|}{19} & \multicolumn{2}{|c|}{23} \\
\hline & & & & $3-\omega$ & $5-\omega$ & $2-\omega$ & $3+\omega$ & $1-\omega$ & $2+\omega$ & $\omega$ & $1+\omega$ & $6-\omega$ & $7+\omega$ \\
\hline$g_{k}(\mathfrak{p})$ & 1 & 2 & $2^{2}$ & 3 & 5 & 1 & 1 & 1 & 1 & 1 & 1 & 1 & 1 \\
\hline 29 & 31 & & & & 11 & 43 & 47 & & 3 & 59 & & & \\
\hline & & $7-\omega$ & $8+\omega$ & $7+2 \omega$ & $5-2 \omega$ & & & $8-\omega$ & $9+\omega$ & & & & \\
\hline $2^{2} \cdot 7$ & $2 \cdot 3 \cdot 5$ & 2 & 2 & 1 & 1 & $2^{2} \cdot 3 \cdot 7$ & $2^{2} \cdot 23$ & 2 & 2 & $2 \cdot 29$ & & & \\
\hline
\end{tabular}

j) $k=Q(\sqrt{93}), \omega=(-1+\sqrt{93}) / 2$, fundamental unit $=(29+3 \sqrt{93}) / 2=16+3 \omega$

\begin{tabular}{|c|c|c|c|c|c|c|c|c|c|c|c|c|}
\hline & 2 & 3 & 5 & \multicolumn{2}{c|}{7} & \multicolumn{2}{|c|}{11} & 13 & \multicolumn{2}{c|}{17} & \multicolumn{2}{c|}{19} \\
\hline & & $4-\omega$ & & $5-\omega$ & $6+\omega$ & $3-\omega$ & $4+\omega$ & & $2-\omega$ & $3+\omega$ & $6-\omega$ & $7+\omega$ \\
\hline$g_{k}(\mathfrak{p})$ & 1 & 1 & $2^{2}$ & 1 & 1 & 1 & 1 & $2^{2} \cdot 3$ & 1 & 1 & 1 & 1 \\
\hline
\end{tabular}




\begin{tabular}{|c|c|c|c|c|c|c|c|c|c|c|c|}
\hline \multicolumn{2}{|c|}{23} & \multicolumn{2}{|c|}{29} & 31 & 37 & 41 & 43 & 47 & 53 & 59 & 61 \\
\hline$\omega$ & $1+\omega$ & $9+2 \omega$ & $7-2 \omega$ & $14-3 \omega$ & & & & & $14+3 \omega$ & $11-3 \omega$ & \\
\hline 1 & 1 & 7 & 7 & $3 \cdot 5$ & $2^{2} \cdot 3^{2}$ & $2^{3} \cdot 5$ & $2^{2} \cdot 3 \cdot 72 \cdot 3 \cdot 23$ & 1 & 1 & $2 \cdot 29$ & $2^{2} \cdot 3 \cdot 5$ \\
\hline
\end{tabular}

\section{$\S 5$. Construction of genus field.}

For a finite abelian group $G$, we denote by $|G|$ the order of $G, \operatorname{ex}(G)$ the exponent of $G$, that is, the smallest positive integer which annihilates $G$. For integer $m$ and $n, n \mid m^{\infty}$ means that $n \mid m^{t}$ for sufficiently large $t$.

THEOREM 8. Let $k$ be a finite algebranc number field of class number one, $G$ any finte abelian group, and $m$ a positive integer such that $e x(G) \mid m$ and $m \|\left. G\right|^{\infty}$. Then there exist infinitely many cyclic extensions $F$ of $k$ of degree $m$ such that

$$
C_{F} / C_{F}^{1-\sigma} \cong G\left(F^{*} / F\right) \cong G
$$

where $C_{F}$ is the ideal class group of $F$ on which $G(F / k)$ acts in usual way and $\sigma$ is one of the generators of cyclic group $G(F / k)$.

To prove this theorem, we use the following lemma proved in [7]. For a rational prime number $l$ and a positive integer $n$, we put

$$
k(l, n)=k\left(\zeta, \varepsilon_{1}^{1 / l^{-n}}, \cdots, \varepsilon_{r}^{1 / l^{-n}}\right)
$$

where $l^{\delta}$ is the number of $l$-power roots of unity in $k, \zeta$ is a primitive $l^{\delta+n}$-th root of unity, and $\left\{\varepsilon_{1}, \cdots, \varepsilon_{r}\right\}$ is the fundamental units of $k$. Then we have

LEMMA 9. Let $k$ be a finte algebranc number field. For a rational prime number $l$ such that $l \times h_{k}$, a positive integer $n$, and a finite prime $\mathfrak{p}$ of $k$, the followng ${ }^{-}$three conditions are equivalent;

1) Let $S$ be the ray class field modulo $\mathfrak{p}$ of $k$. Then there exists an intermediate field $L$ of $S / k$ such that $(L: k)=l^{n}$.

2) $l^{n}$ divides $\phi(\mathfrak{p}) /\left(U_{k}: U_{k}(\mathfrak{p})\right)$.

3) $\mathfrak{p} \nmid l$ and $\mathfrak{p}$ splits completely in $k(l, n)$.

When these three equivalent conditions are fulfilled, $L$ is a cyclic extension of $k$ and $\mathfrak{p}$ is totally ramified in $L$. Therefore the intermediate field of 1) is unique.

Proof of Theorem 8. Suppose first that $G$ is $l$-primary for a rational prime number $l$. Then we have

$$
G=G_{1} \times \cdots \times G_{t}, \quad m=l^{e_{t+1}}
$$

where $G_{\jmath}$ is cyclic group of order $l^{e}$ and $1<e_{1} \leqq, \cdots, \leqq e_{t} \leqq e_{t+1}$. By Lemma 9, 
there exist distinct finite primes $\mathfrak{p}_{1}, \cdots, \mathfrak{p}_{t+1}$ of $k$ such that $l^{e}$ divides $\phi\left(\mathfrak{p}_{j}\right) /\left(U_{k}: U_{k}\left(\mathfrak{p}_{j}\right)\right)$. Let $S_{\jmath}$ be the ray class field modulo $\mathfrak{p}_{\jmath}$ of $k, L$, the intermediate field of $S_{j} / k$ such that $\left(L_{\jmath}: k\right)=l^{e_{3}}$, and $\sigma_{\jmath}$ a generator of cyclic group $G\left(L_{j} / k\right)$. Put $K=\prod_{j=1}^{t+1} L_{\jmath}$ composite field, then we have

$$
G(K / k)=G\left(L_{1} / k\right) \times \cdots \times G\left(L_{t+1} / k\right) .
$$

Let $H$ be the subgroup of $G(K / k)$ generated by $\left\{\sigma_{j} \sigma_{t+1}^{l^{n-e} \jmath}:(1 \leqq \jmath \leqq t)\right\}$, and $F$ the fixed field of $H$ (the construction of $F$ is due to [7]). Then $G(F / k)=G(K / k) / H$ is a cyclic group of order $m$ whose generator is $\sigma_{t+1} H$, and $\sigma$, generates the inertia group of $\mathfrak{p}_{\jmath}$ in $K / k$, for $1 \leqq \jmath \leqq t+1$. Therefore $K$ is unramified over $F$, and hence $K \subset F^{*}$. Since $h_{k}=1$, we have $(K: k) \geqq\left(F^{*}: k\right)$ by the genus number formula, and hence $K=F^{*}$. Then we have

$$
G\left(F^{*} / F\right)=H \cong G_{1} \times \cdots \times G_{t}=G .
$$

For general $G$, we have

$$
G=G_{1} \times \cdots \times G_{s}, \quad m=q_{1} \cdots q_{s}
$$

where $G_{\rho}$ is the $l_{\jmath}$-primary part of $G$ for a rational prime number $l_{\jmath}$, and $q_{\jmath}$ is a power of $l_{\jmath}$. Then there exists a cyclic extension $F_{\jmath}$ of $k$ of degree $q_{j}$ such that

$$
G\left(F_{\jmath}^{*} / F_{\jmath}\right) \cong G_{\jmath} .
$$

Let $F=\prod_{\jmath=1}^{s} F_{\jmath}$ composite field. Then, by Lemma 6 , we have $F^{*}=\prod_{\jmath=1}^{s} F_{\jmath}^{*}$ composite field. By the genus number formula, $\left\{\left(F_{j}^{*}: k\right):(1 \leqq \jmath \leqq s)\right\}$ are mutually prime, therefore we have

$$
G\left(F^{*} / F\right) \cong G\left(F_{1}^{*} / F_{1}\right) \times \cdots \times G\left(F_{s}^{*} / F_{s}\right) \cong G_{1} \times \cdots \times G_{s}=G .
$$

The infinity of $F$ is follows from the way of construction of $F$ and from Lemma 9. The fact that the Artin mapping gives the isomorphism $C_{F} / C_{F}^{1-\sigma}$ $\cong G\left(F^{*} / F\right)$ is proved in [8].

\section{REFERENCES}

[1] M. Bhaskaran, Construction of genus field and some applications. J. Number Theory 11 (1979), 488-497.

[2] Y. Furuta, The genus field and genus number in algebraic number field. Nagoya Math. J. 29 (1967), 281-285.

[3] R. Gold and L. MAdAN, Some applications of Abhyanker's lemma. Math. Nachr. 82 (1978), 115-119.

[4] M. IsHidA, Some unramified abelian extensions of algebraic number field. J. Reine Angew. Math. 268/269 (1974), 165-173.

[5] M. IsHidA, The genus fields of algebraic number fields. Springer Lecture Note 555 (1976). 
[6] M. IsHidA, On the genus fields of pure nuumber fields. Tokyo J. Math. Vol. 3 No. 1 (1980), 163-171.

[7] O. YAHAGI, Construction of number fields with prescribed $l$-class group. Tokyo J. Math. Vol. 1 No. 2 (1978), 275-283.

[8] H. Yokol, On the class number of relatively cyclic number field. Nagoya Math. J. 29 (1967), 31-44.

Department of Mathematics

TOKYo Institute of TECHNOLOGY 